\title{
Identificación de puntos de referencia anatómicos para la valoración del equilibrio mediante dispositivos cinemáticos
}

\author{
Identification of anatomical reference points to assess balance using kinematic devices
}

\author{
R. Leirós-Rodríguez ${ }^{\mathrm{a}}$, M.E. Arce ${ }^{\mathrm{b}}$, A. Souto-Gestal ${ }^{\mathrm{c}}$, J.L. García-Soidán ${ }^{\mathrm{a}}$ \\ ${ }^{a}$ Departamento de Didácticas Especiales, Facultad de Ciencias de la Educación y del Deporte, Universidad de Vigo, \\ Pontevedra, España \\ ${ }^{b}$ Centro Universitario de la Defensa, Universidad de Vigo, Marín, Pontevedra, España \\ ${ }^{c}$ Departamento de Fisioterapia, Facultad de Fisioterapia, Universidad de A Coruña, A Coruña, España
}

\begin{abstract}
Resumen
Objetivo. Analizar el comportamiento de los niveles raquídeos más empleados en el análisis cinemático y definir el punto óptimo de colocación de acelerómetros para la medida del equilibrio en adultos.

Material y método. Se valoró a un total de 71 sujetos sanos de los que la mayoría fueron mujeres $(92,96 \%)$, con una edad media \pm desviación estándar de 64,86 \pm 7,63 años. Para la valoración se utilizaron 3 acelerómetros triaxiales ubicados en esternón, L4 y L5-S1, que registraron datos cinemáticos durante la realización de 2 pruebas de equilibrio: equilibrio monopodal con ojos cerrados y equilibrio dinámico monopodal.

Se consideraron criterios de exclusión para participar en el estudio la incapacidad para realizar la marcha de forma independiente, para mantener el equilibrio estático bipodal con ojos abiertos durante $60 \mathrm{~s}$ o la presencia de alguna contraindicación. Todos los sujetos realizaron las pruebas descalzos con calcetines.

Resultados. Se encontró que las oscilaciones producidas en las 3 ubicaciones de referencia analizadas durante las pruebas de equilibrio se incrementan de forma proporcional a la distancia al centro de gravedad. En relación con el punto óptimo de colocación del dispositivo en la región lumbo-pélvica, los resultados obtenidos indicaron que no existen diferencias relevantes entre ubicar el dispositivo al nivel de L4 o L5-S1.

Conclusiones. Se recomienda la elección de la cuarta vértebra lumbar como punto de medición, ya que así se limita la introducción de ruido en la señal recogida por los acelerómetros derivada de la movilidad intrínseca de la cintura pélvica.
\end{abstract}

\begin{abstract}
Objective. This paper has aimed to analyze the behavior of the spinal levels used most in the kinematic analysis and to define the best place for accelerometers to measure balance in adults.

Material and method. A total of 71 healthy adults were evaluated. Most were women $(92.96 \%)$, with an average age of 64.86 years $(\mathrm{SD} \pm 7.63)$. Three tri-axial accelerometers were used to evaluate balance. One was placed on the sternum and two more on L4 and L5-S1. Kinematic data was obtained from two balance tests: eyes closed monopodal balance and monopodal dynamic balance.

Exclusion criteria included inability to walk independently; to be unable to maintain bipodal static balance with eyes wide-open for 60 seconds and the existence of contraindications to be tested. All the subjects performed the tests in bare feet with socks.

Results. The results showed that the three reference locations analyzed during the balance tests proportionally increased to the distance to the center of gravity. Regarding the optimal devise placement point in the lumbar-pelvic regions, the results obtained did not show significant differences between the accelerometer on L4 and L5-S1.

Conclusions. Using the fourth lumbar vertebra for measurement is recommended because it limits the introduction of noise on the accelerometer signal due to pelvic girdle motion.
\end{abstract}

Palabras clave

Acelerometría; Equilibrio; Cinemática; Centro de masas; Antropometría

Keywords

Accelerometry; Balance; Kinematics; Center of mass; Anthropometry 


\section{Introducción}

La utilización de acelerómetros para la valoración del equilibrio se ha visto limitada por la falta de protocolos que permitan estandarizar su aplicación. La literatura científica se ha focalizado en la justificación de la validez de estos instrumentos, tanto desde el punto de vista biomecánico, como fisiológico ${ }^{1,2}$ and 3 . De tal forma que la búsqueda de la optimización y la estandarización de su modo de empleo ha quedado en un segundo plano $1,2,3,4$ and 5 .

En relación con el uso de acelerómetros en el ámbito clínico, existen tantas metodologías que incluso un aspecto fundamental como la ubicación del dispositivo durante las pruebas llega a diferir significativamente entre los distintos trabajos, incluso en aquellos que tienen, a priori, un mismo objetivo de medición ${ }^{5}$. Sin embargo, existe suficiente evidencia empírica que señala que la colocación de los dispositivos sobre el centro de gravedad $(\mathrm{CG})$ es la que mejor refleja el funcionamiento del sistema de control postural y sus estrategias de estabilización ${ }^{6}$ and 7 .

La elección del CG como punto de referencia de las estrategias de estabilización y reacciones de enderezamiento se basa en el análisis del cuerpo en su totalidad ${ }^{7}$. Este enfoque asume que el control postural es una aptitud fruto de la integración de diferentes regiones corporales dirigidas y coordinadas por el sistema nervioso central (SNC), y a partir del cual se ha desarrollado la teoría multisegmentaria del equilibrio $^{7 \text { and } 8}$.

Así, una vez definido el CG como localización de referencia para la valoración del equilibrio, es necesario saber dónde ubicarlo en el cuerpo del individuo. Al no existir un criterio único, nos encontramos con que se toman como referencia del centro de masas distintos niveles del raquis, siendo los más utilizados la tercera y la cuarta vértebra lumbar y la base sacra ${ }^{9}$.

Por otra parte, resulta importante destacar que, aunque la ubicación exacta del CG en el cuerpo ha sido objeto de estudio dentro del ámbito científico, hasta el momento no se ha establecido un punto o rango de puntos de medición estándar. Esto es debido a que la mayoría de las investigaciones se centraron en determinar la posición del centro de masas de forma individualizada, y no a obtener un valor genérico que pueda extrapolarse a diferentes individuos. Además, estos trabajos se valen de complejos equipos dotados

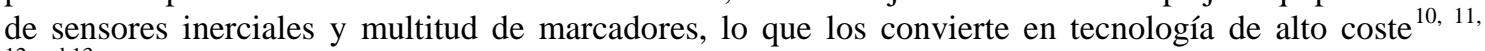
12 and 13

En la práctica clínica, estos procesos no son factibles, dado su elevado coste económico y la dificultad de montaje, ajuste e interpretación. Todo ello limita la utilización y aplicabilidad de la cinemática como método de valoración del equilibrio en el ámbito asistencial. De este modo, se ha llevado a cabo la presente investigación, en la que se compara el comportamiento de los niveles raquídeos más empleados en análisis cinemático con el objetivo de definir el punto óptimo de colocación de los dispositivos de medida.

\section{Material y métodos}

\section{Participantes}

Se valoró a un total de 71 sujetos, de los que la mayoría fueron mujeres $(92,96 \%)$, con una edad media \pm desviación estándar de 64,89 \pm 7,63 años. Todos los participantes firmaron el consentimiento informado, antes de su intervención en el estudio, de acuerdo con la declaración de Helsinki (rev. 2008). Con el objeto de homogeneizar las condiciones de realización de las pruebas, todos los sujetos debían vestir ropa cómoda y permanecer descalzos con calcetines.

Se consideraron criterios de exclusión para la participación en este estudio la incapacidad para realizar la marcha de forma independiente o sin elementos ortopédicos externos, para mantener el equilibrio estático bipodal con ojos abiertos durante al menos $60 \mathrm{~s}$ o la presencia de alguna contraindicación para someterse a alguna de las pruebas de valoración.

\section{Instrumentos de medición}

Para la medición cinemática de las aceleraciones producidas se utilizaron acelerómetros triaxiales (modelo ActiGraph GT3X-plus). Estos dispositivos permiten almacenar series temporales de datos de aceleración en una memoria flash no volátil. Las pequeñas dimensiones de los módulos $(4,6 \times 3,3 \times 1,5 \mathrm{~cm})$, unidas a su poco peso $(19 \mathrm{~g})$, hacen de estos dispositivos una de las mejores 
opciones para evaluar cambios en la posición corporal. La precisión de los acelerómetros es de $3 \mathrm{mg} / \mathrm{LSB}$ y su rango de medición de $\pm 6 \mathrm{G}$.

Los acelerómetros proporcionan los datos de las aceleraciones en los 3 ejes, siendo:

- el eje 1 el que representa la aceleración en el eje vertical (y, equivalente al eje cráneo-caudal);

- el eje 2 reflejo de la aceleración en el eje horizontal y parejo al eje medio-lateral,

- el eje 3, representación de la aceleración en el eje perpendicular o antero-posterior.

Puesto que en las acelerometrías solo interesa detectar movimientos que se puedan atribuir a actividad humana y no a vibraciones menores del entorno, la señal detectada por el acelerómetro ha de pasar un filtro antes de ser procesada. Este umbral hace que la capacidad de medición de los dispositivos se vea limitada, especialmente cuando se trata de analizar poblaciones de personas de avanzada edad, en las que los movimientos son de menor frecuencia y mayor amplitud. Por ello, la frecuencia de trabajo seleccionada fue $30 \mathrm{~Hz}$. Además, al trabajar con una baja frecuencia, se logra eliminar de manera más efectiva el ruido de la señal.

Se ha de tener en consideración que durante la toma de datos un mismo acelerómetro se utiliza con diferentes sujetos, por lo que en la etapa de posprocesado de datos es fundamental conocer cuándo el acelerómetro le fue retirado a cada sujeto. Por ello, los acelerómetros a utilizar deben contar con un inclinómetro que permita conocer la posición del sujeto y si el aparato se está utilizando o no. Los resultados del inclinómetro servirán para verificar los datos de las acelerometrías.

Como instrumento de comprobación adicional, se utilizó una cámara de vídeo que registró la realización de todas las pruebas. Este elemento permitió obtener una visión posterior del sujeto. Las grabaciones fueron analizadas en la etapa de posprocesado, lo que permitió identificar cualitativamente las estrategias de estabilización de los sujetos y determinar cómo estas se reflejan a nivel cuantitativo en las aceleraciones.

La cámara se situó a $3 \mathrm{~m}$ de distancia del punto donde se encontraban los sujetos realizando las pruebas de equilibrio. Esta se fijó sobre un trípode, de tal forma que permitiese modificar la altura en función de las dimensiones de la persona de estudio de modo que fuese posible capturar una imagen del cuerpo completo.

\section{Protocolo de medición}

Se utilizaron 3 equipos colocados directamente sobre la piel (fig. 1):

- En la espalda, a la altura de la apófisis espinosa de la cuarta vértebra lumbar.

- En la pelvis, a la altura de la charnela lumbo-sacra (entre la espinosa de la apófisis espinosa de la quinta vértebra lumbar y la base sacra).

- En la cara anterior del tronco, sobre el esternón, a la altura de la articulación costo-esternal correspondiente la quinta costilla. 

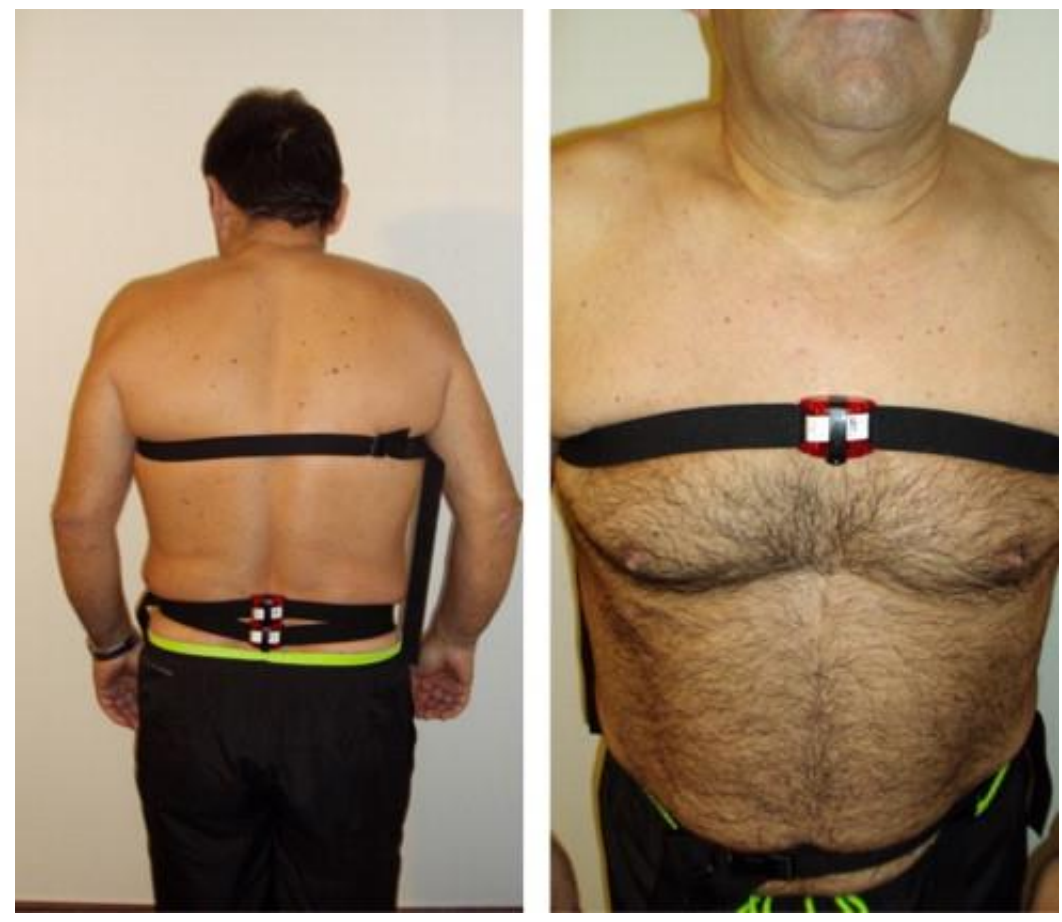

Figura 1. Ubicación de los acelerómetros durante la realización de las pruebas.

La fijación de los mismos sobre la piel se aseguró con cinturones ajustables y cinta adhesiva hipoalergénica para cerciorar que el aparato no se movía independientemente del tronco del sujeto durante la realización de las pruebas.

Los participantes debían realizar 2 pruebas: una en equilibrio monopodal con ojos cerrados y otra en equilibrio dinámico monopodal. Todas las pruebas tuvieron una duración de $30 \mathrm{~s}$ y se repitieron 3 veces, con descansos de $10 \mathrm{~s}$ entre cada una de las mismas. Durante las pruebas de evaluación, todos los participantes recibieron instrucciones de que su objetivo era permanecer lo más quietos posible manteniendo la postura erguida, ante las diferentes condiciones de valoración que se les propusieran. Asimismo, se les solicitó que mantuviesen los brazos cruzados sobre el pecho y con la mirada al frente.

Se seleccionaron estas 2 pruebas de entre un total de 6 test de equilibrio analizados en un estudio piloto con 20 sujetos $^{14}$, debido a que en ellas se encontró un mayor registro de oscilaciones. El equilibrio estático fue valorado directamente sobre el suelo, a través de la prueba de equilibrio monopodal con ojos cerrados, mientras que el equilibrio dinámico se analizó sobre una superficie inestable (en nuestro caso, una colchoneta de gimnasia estándar) a través de la prueba de equilibrio monopodal con ojos abiertos (fig. 2). Todas las colchonetas utilizadas tenían las mismas características: material base de etilvinilacetato (goma EVA), cubierta de vinilo y base antideslizante. 

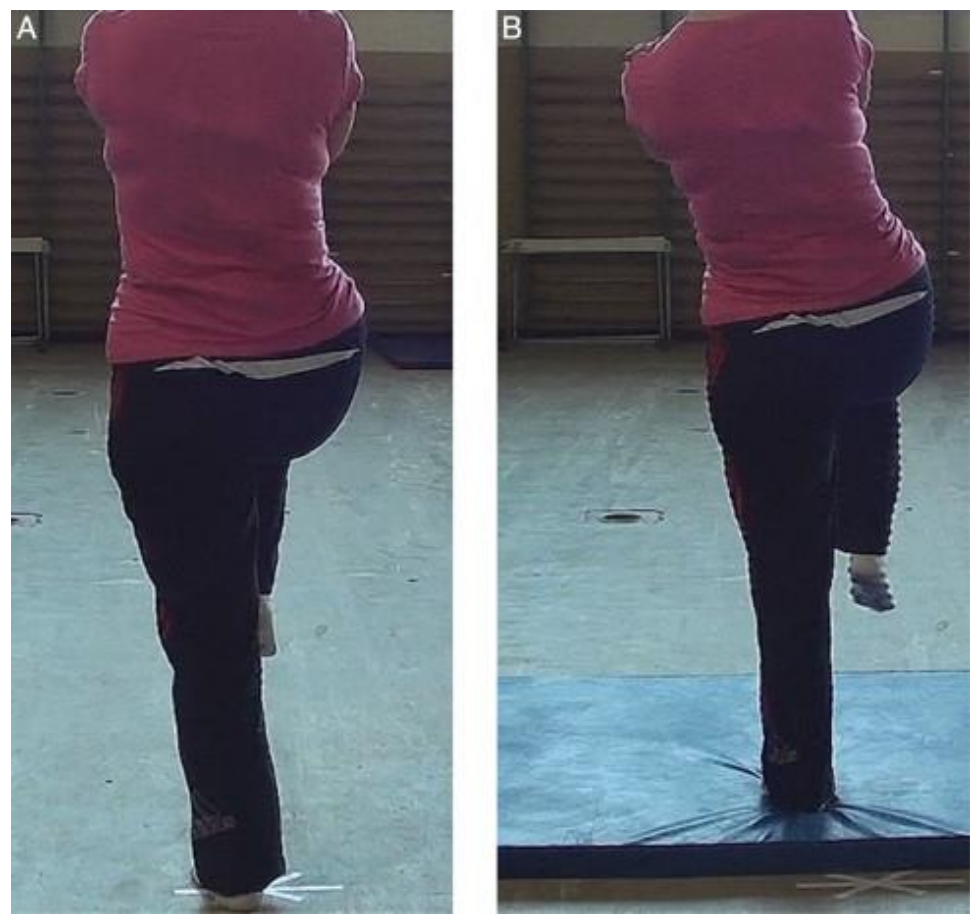

Figura 2. Sujeto durante la realización de la prueba de equilibrio monopodal con ojos cerrados (A) y equilibrio dinámico monopodal (B).

Se permitió al sujeto elegir sobre qué pierna realizar el apoyo, de tal forma que el participante pudo hacer ensayos antes de comenzar la valoración, y así decidir sobre qué miembro realizaría todas las pruebas.

\section{Resultados}

A continuación, se describen los resultados obtenidos de los 50.514 registros analizados tras procesar las señales de los acelerómetros en las mediciones de cada una de las 3 series de las pruebas. La cantidad de datos sometida a análisis se multiplicó por 3 debido a que se tomaron 3 puntos de medición para cada sujeto y prueba.

\section{Equilibrio dinámico}

Los resultados obtenidos mostraron una correlación significativa entre la edad y la aceleración promedio en la prueba de equilibrio monopodal dinámico para los acelerómetros ubicados tanto en L5-S1, $r=481, p<0,01$, como en L4, r = 70, p < 01 y en el esternón, $r=421, p<0,01$ (tabla 1). Asimismo, los datos obtenidos con dispositivos ubicados en L5-S1 correlacionaron con los obtenidos con acelerómetros ubicados en L4, r = 982 p <01; y en el esternón, $r=838 \mathrm{p}<01$, es decir, las variaciones posturales obtenidas con dispositivos ubicados en L5-S1 explican el 96,63 y el 70,22\% de los resultados obtenidos con acelerómetros ubicados en L4 y el esternón, respectivamente. 
Tabla 1. Resultados análisis correlaciones bivariadas para la prueba de equilibrio dinámico monopodal

\begin{tabular}{|c|c|c|c|c|c|}
\hline & & Edad & $\begin{array}{l}\text { Ac. ubicado en L5-S1 } \\
\text { Módulo vector } \\
\text { aceleración }\end{array}$ & $\begin{array}{l}\text { Ac. ubicado en L4 } \\
\text { Módulo vector } \\
\text { aceleración }\end{array}$ & $\begin{array}{l}\text { Ac. ubicado en esternón } \\
\text { Módulo vector } \\
\text { aceleración }\end{array}$ \\
\hline \multirow[t]{2}{*}{ Edad } & $\begin{array}{l}\text { Correlación de } \\
\text { Pearson }\end{array}$ & 1 & $0,481^{\mathrm{a}}$ & $0,470^{\mathrm{a}}$ & $0,421^{\mathrm{a}}$ \\
\hline & Sig. (bilateral) & & 0,000 & 0,000 & 0,000 \\
\hline \multirow[t]{2}{*}{$\begin{array}{l}\text { Acelerómetro ubicado en L5- } \\
\text { S1 } \\
\text { Módulo vector aceleración }\end{array}$} & $\begin{array}{l}\text { Correlación de } \\
\text { Pearson }\end{array}$ & $0,481^{\mathrm{a}}$ & 1 & $0,982^{\mathrm{a}}$ & $0,838^{\mathrm{a}}$ \\
\hline & Sig. (bilateral) & 0,000 & & 0,000 & 0,000 \\
\hline \multirow[t]{2}{*}{$\begin{array}{l}\text { Acelerómetro ubicado en L4 } \\
\text { Módulo vector aceleración }\end{array}$} & $\begin{array}{l}\text { Correlación de } \\
\text { Pearson }\end{array}$ & $0,470^{\mathrm{a}}$ & $0,982^{\mathrm{a}}$ & 1 & $0,838^{\mathrm{a}}$ \\
\hline & Sig. (bilateral) & 0,000 & 0,000 & & 0,000 \\
\hline \multirow[t]{2}{*}{$\begin{array}{l}\text { Acelerómetro ubicado en } \\
\text { Esternón } \\
\text { Módulo vector aceleración }\end{array}$} & $\begin{array}{l}\text { Correlación de } \\
\text { Pearson }\end{array}$ & $0,421^{\mathrm{a}}$ & $0,838^{\mathrm{a}}$ & $0,838^{\mathrm{a}}$ & 1 \\
\hline & Sig. (bilateral) & 0,000 & 0,000 & 0,000 & \\
\hline
\end{tabular}

Al analizar las aceleraciones medias en cada prueba en cada eje y el vector de módulo resultante, se observó un incremento del vector aceleración en la segunda y la tercera serie de la prueba (tabla 2). Estos resultados evidencian la existencia de un efecto fatiga en los participantes a lo largo de las 3 series de repeticiones.

Tabla 2. Aceleraciones medias. Resultados del análisis de los acelerómetros ubicados en L5-S1

\begin{tabular}{|c|c|c|c|c|c|}
\hline Prueba & Serie & $\begin{array}{l}\text { Aceleración media } \\
\text { Eje } 1\end{array}$ & $\begin{array}{l}\text { Aceleración media } \\
\text { Eje } 2\end{array}$ & $\begin{array}{l}\text { Aceleración media } \\
\text { Eje } 3\end{array}$ & $\begin{array}{l}\text { Aceleración media } \\
\text { Módulo vector aceleración }\end{array}$ \\
\hline \multirow[t]{3}{*}{ Equilibrio monopodal ojos cerrados } & 1 & $1,73 \mathrm{G}$ & $6,47 \mathrm{G}$ & $3,69 \mathrm{G}$ & $8,88 \mathrm{G}$ \\
\hline & 2 & $2,36 \mathrm{G}$ & $6,50 \mathrm{G}$ & $4,45 \mathrm{G}$ & $9,84 \mathrm{G}$ \\
\hline & 3 & $2,49 \mathrm{G}$ & $7,24 \mathrm{G}$ & $5,01 \mathrm{G}$ & $10,76 \mathrm{G}$ \\
\hline \multirow[t]{3}{*}{ Equilibrio dinámico monopodal } & 1 & $0,94 \mathrm{G}$ & $4,04 \mathrm{G}$ & $2,43 \mathrm{G}$ & $5,56 \mathrm{G}$ \\
\hline & 2 & $1,35 \mathrm{G}$ & $4,65 \mathrm{G}$ & $3,31 \mathrm{G}$ & $6,94 \mathrm{G}$ \\
\hline & 3 & $1,89 \mathrm{G}$ & $4,99 \mathrm{G}$ & $3,74 \mathrm{G}$ & $7,76 \mathrm{G}$ \\
\hline
\end{tabular}

\section{Equilibrio estático}

Al comparar las mediciones cinemáticas registradas por cada uno de los 3 dispositivos no se obtuvo ningún patrón de comportamiento en lo que refiere al desarrollo de la tarea.

Sin embargo, cuando se analizó el tiempo en el que se produjeron los picos aceleración, se observó que los datos obtenidos con los dispositivos ubicados a la altura de la L4 y L5-S1 marcaron tiempos muy similares (tabla 3), lo que indicó que los datos obtenidos con ambos instrumentos eran redundantes, resultado que confirmó lo hallado en la prueba de equilibrio dinámico. Conviene señalar que las aceleraciones máximas se produjeron en los segundos centrales de la prueba, con independencia de la serie o prueba que se estuviera realizando, tanto para equilibrio monopodal ojos cerrados, como para equilibrio dinámico monopodal (fig. 3). 
Tabla 3. Tiempo en el que se producen las aceleraciones pico en cada uno de los 3 ejes y para cada dispositivo como media de las 3 pruebas

Segundo en el que se produce la variación de velocidad máxima

\begin{tabular}{|c|c|c|c|c|}
\hline & Eje 1 & Eje 2 & Eje 3 & \\
\hline \multirow[t]{2}{*}{ Dispositivo situado en L5-S1 } & Media & 15,31 & 17,35 & 19,18 \\
\hline & Desviación típica & 8,29 & 9,42 & 8,25 \\
\hline \multirow[t]{2}{*}{ Dispositivo situado en L4 } & Media & 15,18 & 17,64 & 19,09 \\
\hline & Desviación típica & 8,57 & 9,29 & 8,71 \\
\hline \multirow[t]{2}{*}{ Dispositivo situado en esternón } & Media & 19,43 & 18,06 & 20,34 \\
\hline & Desviación típica & 9,14 & 8,77 & 9,03 \\
\hline
\end{tabular}

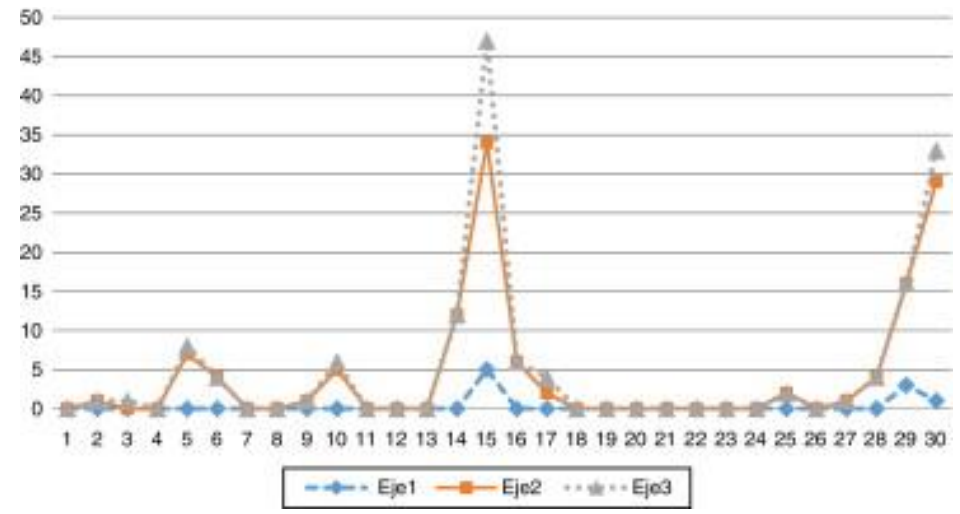

Figura 3. Variaciones de velocidad obtenidas en los 3 ejes y módulo vector de aceleración para un sujeto a lo largo de la prueba de equilibrio monopodal con ojos cerrados.

Tal y como se mencionó en secciones anteriores, además de comparar los resultados cinemáticos obtenidos en las diferentes pruebas, se realizó un estudio de las imágenes recogidas en vídeo. Esto permitió determinar el umbral a partir del cual existe una pérdida irreversible del equilibrio, es decir, la necesidad de pasar a posición bípeda o, incluso, una caída.

Del análisis de imágenes, se pudieron clasificar las alteraciones detectadas en las siguientes:

- Inclinaciones de tronco (izquierda y derecha).

- Flexiones de tronco.

- Abducciones y aducciones de cadera.

- Saltos reequilibrantes (saltos sobre el pie de apoyo para recuperar la base de sustentación).

- Pérdida irreversible del equilibrio (p. el., apoyo de pie, pérdida total de la base de sustentación).

Una vez identificado el significado de las alteraciones, se procedió a determinar el umbral acelerométrico para el cual se producía el desequilibrio en los 3 puntos de medición, tomándose como parámetros de corte las alteraciones que suponían pérdida irreversible de desequilibrio. El eje en el que se detectaron las mayores aceleraciones fue el anteroposterior, siendo el valor umbral de $18 \pm 15$ G. Por su parte, el eje cráneo caudal, que es en el que menores variaciones se encuentran, muestra un valor umbral de $5 \pm 10 \mathrm{G}$ y en el eje lateromedial de $10 \pm 12 \mathrm{G}$. El valor umbral acelerométrico para el que tenemos alteraciones del equilibrio del vector resultante (valor cuadrático medio) se fijó en $24 \pm 19 \mathrm{G}$. Las pruebas en las que se produjo un mayor número de alteraciones fue la de equilibrio monopodal con ojos cerrados, es decir, aquella en la que el sujeto carecía de información visual. El efecto fatiga se observó tanto en las 
pruebas estáticas como dinámicas, detectando un mayor número de casos de pérdidas del equilibrio en la tercera serie con respecto a las 2 primeras.

\section{Discusión}

Este trabajo tenía como objetivo la determinación del punto óptimo de colocación de acelerómetros en relación con el CG ya que, aunque se estima que este se encuentra en la cintura pélvica, no existen estudios que hayan realizado una comparación de los 2 niveles lumbo-pélvicos más empleados para ubicar este tipo de dispositivos de medición. A la luz de los resultados expuestos, se ha evidenciado que incluso con dispositivos situados a distancia del CG existe una alta correspondencia entre los registros obtenidos en las diferentes ubicaciones.

Como valor añadido, en este estudio, se triangularon los datos cinemáticos con la visualización de vídeos de las pruebas de equilibrio. Este análisis cruzado de los datos permitió dotar de significado cualitativo las aceleraciones detectadas, haciendo así más fiables los resultados.

Ahora bien, siendo conscientes de que los datos obtenidos por los dispositivos de medición cinemática mostraron una elevada correlación, en ningún caso debe concluirse que todos transmitan la misma información o que se puedan utilizar las aceleraciones de un solo segmento en representación del conjunto del cuerpo.

Puesto que se trataba de una muestra formada por individuos sanos, es natural que las estrategias de estabilización empleadas durante las pruebas estudiadas se desarrollen de forma armónica en todo el tronco. Es decir, que los dispositivos mostrasen un patrón de comportamiento proporcional en los diferentes puntos del tronco indica la ausencia de deterioro de los sistemas de control postural ${ }^{15}$. Sin embargo, aún no se ha determinado si existe alguna relación entre los cambios en las aceleraciones de otras partes del cuerpo y el riesgo de caída o inestabilidad postural, habiendo solo evidencia de la fiabilidad de las medidas tomadas en la cintura pélvica ${ }^{16}$ and 17.

\section{Conclusiones}

En relación con la elección del punto óptimo de colocación de dispositivo en la región lumbo-pélvica, dado que los resultados obtenidos por los 2 dispositivos ubicados en la misma no detectaron diferencias estadísticamente significativas, se recomienda atender a razones biomecánicas para la toma de esta decisión. Por tanto, la posibilidad de que los movimientos específicos de la cintura pélvica, anteversión y retroversión, puedan introducir ruido en los resultados de las aceleraciones detectadas por un dispositivo sobre la base sacra motiva que se considere el cuarto nivel lumbar como punto de registro cinemático más adecuado.

Del mismo modo, asumimos la limitación del trabajo en cuanto a que los resultados obtenidos no permiten describir desde un punto de vista fisiológico cómo funcionan los sistemas de control postural para el mantenimiento del equilibrio, siendo estos solo una medida indirecta de la eficacia con la que el SNC integra la información del entorno y del propio sujeto con el objetivo de mantener el equilibrio.

Finalmente, debemos destacar la relevancia de haber identificado la ubicación óptima en el tronco de dispositivos cinemáticos con tecnología de bajo coste para evaluar el equilibrio en adultos sanos, sin disfunciones sensorio-perceptivo-motrices. Además, se ha obtenido un umbral cuantitativo a partir del cual el riesgo de caída podría resultar clínicamente relevante, por lo que presenta una gran aplicabilidad en las pruebas de cribado empleadas el ámbito asistencial o en la evaluación de la eficacia de los programas de prevención de caídas ${ }^{18}$ and 19 .

\section{Responsabilidades éticas}

\section{Protección de personas y animales}

Los autores declaran que los procedimientos seguidos se conformaron a las normas éticas del comité de experimentación humana responsable y de acuerdo con la Asociación Médica Mundial y la Declaración de Helsinki.

\section{Confidencialidad de los datos}

Los autores declaran que en este artículo no aparecen datos de pacientes. 


\section{Derecho a la privacidad y consentimiento informado}

Los autores han obtenido el consentimiento informado de los pacientes y/o sujetos referidos en el artículo. Este documento obra en poder del autor de correspondencia.

\section{Conflictos de intereses}

Los autores declaran la no existencia de conflictos de intereses.

\section{Bibliografía}

1. O. Aziz, E. Park, G. Mori, S. Robinovitch. Distinguishing the causes of falls in humans using an array of wearable tri-axial accelerometers. Gait Posture., 39 (2014), pp. 506-512.

2. S. Whitney, J. Roche, G. Marchetti, C. Lin, D. Steed, G. Furman, et al. A comparison of accelerometry and center of pressure measures during computerized dynamic posturography: A measure of balance. Gait Posture., 33 (2011), pp. 594-599.

3. A. Cuesta-Vargas, J. William. Estudio de la cinemática y fiabilidad inter e intraterapeutas de la manipulacion vertebral cervical basada en sensores inerciales. Fisioterapia., 33 (2011), pp. 25-30.

4. A. Hartmann, K. Murer, R.A. de Bie, E.D. de Bruin. Reproducibility of spatio-temporal gait parameters under different conditions in older adults using a triaxial accelerometer system. Gait Posture., 30 (2009), pp. 351-355.

5. A. Hartmann, S. Luzi, K. Murer, R. de Bie, E.D. de Bruin. Concurrent validity of a trunk triaxial accelerometer system for gait analysis in older adults. Gait Posture., 29 (2009), pp. 444-448.

6. A. Adlerton, U. Moritz, R. Moe-Nilssen. Forceplate and accelerometer measures for evaluating the effect of muscle fatigue on postural control during one-legged stance. Physiother Res Int., 8 (2003), pp. 187-199.

7. R. Moe-Nilssen. A new method for evaluating motor control in gait under real-life environmental conditions. Part 1: The instrument. Clin Biomech., 13 (1998), pp. 320-327.

8. P. Hodges, V. Gurfinkel, S. Brumagne, T. Smith, P. Cordo. Coexistence of stability and mobility in postural control: evidence from postural compensation for respiration. Exp Brain Res., 144 (2002), pp. 293-302.

9. Y. Lin, M. Gfoehler, M. Pandy. Quantitative evaluation of the major determinants of human gait. J Biomech., 47 (2014), pp. 1324-1331.

10. K. Lowry, A. Smiley-Oyen, A. Carrel, J. Kerr. Walking stability using harmonic ratios in Parkinson's disease. Mov Disord, 24 (2009), pp. 261-267.

11. Y. Kuramatsu, T. Muraki, Y. Oouchida, Y. Sekiguchi, S. Izumi. Influence of constrained visual and somatic senses on controlling centre of mass during sit-to-stand. Gait Posture., 36 (2012), pp. 90-94.

12. C. Forsell, K. Halvorsen. A method for determining minimal sets of markers for the estimation of center of mass, linear and angular momentum. J Biomech., 42 (2009), pp. 361-365.

13. P. Esser, H. Dawes, J. Collett, K.I.M.U. Howells. Inertial sensing of vertical CoM movement. J Biomech., 42 (2009), pp. 1578-1581.

14. Leirós R, Arce ME, Soidán JL. Estudio preliminar para valoración del equilibrio con un elemento cinemático. X Congreso Internacional de Ciencias del Deporte y la Educación Física. Pontevedra (España). 2014, 8-10 mayo.

15. M. Tucker, J. Kavanagh, R. Barrett, S. Morrison. Age-related differences in postural reaction time and coordination during voluntary sway movements. Hum Movement Sci, 27 (2008), pp. 728-737.

16. S. Mesure, J. Azulay, J. Pouget, B. Amblard. Strategies of segmental stabilization during gait in Parkinson's disease. Exp Brain Res., 129 (1999), pp. 573-581.

17. M. Latt, H. Menz, V. Fung, S. Lord. Acceleration patterns of the head and pelvis during gait in older people with Parkinson's Disease: A comparison of fallers and nonfallers. J Gerontol A Biol Sci Med., 64 (2009), pp. 700-706.

18. J.R. Saiz-Llamosas, V. Casado-Vicente, H.C. Martos-Álvarez. Impacto de un programa de fisioterapia en atención primaria en las personas mayores, con antecedentes de caídas. Fisioterapia., 36 (2014), pp. 103-109.

19. A.M. Martín-Nogueras, J.I. Calvo-Arenillas, M.C. Sánchez-Sánchez, R. Méndez-Sánchez, F.J. Barbero-Iglesias. Incidencia de caídas en mujeres que participan periódicamente en un programa de revitalización geriátrica. Estudio con seguimiento de 5 años. Fisioterapia., 35 (2013), pp. 139-145. 